PREPARED FOR SUBMission TO JINST

\title{
Design of Guide Tube Calibration System for JUNO Experiment
}

\author{
Yuhang Guo, ${ }^{a}$ Qingmin Zhang, ${ }^{a, 1}$ Feiyang Zhang, ${ }^{b}$ Mengjiao Xiao,,${ }^{c, d}$ Jianglai Liu, ${ }^{b}$ Eryuan \\ $\mathbf{Q u}^{a}$ \\ ${ }^{a}$ School of Nuclear Science and Technology, Xi'an Jiaotong University, Xi'an 710049, China \\ ${ }^{b}$ School of Physics and Astronomy, Shanghai Jiao Tong University, Shanghai 200240, China \\ ${ }^{c}$ Department of Physics, University of Maryland, College Park, Maryland 20742, USA \\ ${ }^{d}$ Center of High Energy Physics, Peking University, Beijing 100871, China \\ E-mail: zhangqingmin@mail.xjtu.edu.cn
}

\begin{abstract}
Jiangmen Underground Neutrino Observatory (JUNO) is designed to determine the neutrino mass hierarchy using a 20 kton liquid scintillator detector. To calibrate detector boundary effect, the Guide Tube Calibration System (GTCS) has been designed to deploy a radioactive source along a given longitude on the outer surface of the detector. In this paper, we studied the physics case of this system via simulation, which leads to a mechanical design.
\end{abstract}

Keywords: JUNO, Calibration, Guide Tube, Energy Reconstruction, Non-uniformity Correction

${ }^{1}$ Corresponding author. 


\section{Contents}

1 Introduction 1

2 Physics Considerations of the GTCS Design 3

2.1 Outside or Inside CD? 3

2.2 Choosing the Path of the Guide Tube 6

2.3 Estimation of Uncertainty 6

3 GTCS Mechanical Design 9

3.1 Source and Cable Assembly 9

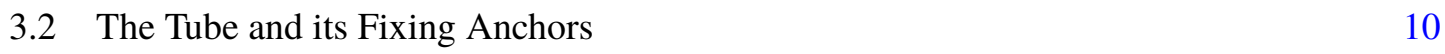

$\begin{array}{ll}\text { 3.3 Winding Machine and its Control System } & 11\end{array}$

4 Summary 12

\section{Introduction}

The Jiangmen Underground Neutrino Observatory (JUNO) is a reactor anti-neutrino experiment currently under construction in Kaiping, China, $53 \mathrm{~km}$ away from both the Taishan and Yangjiang Nuclear Power Plants in Guangdong Province. The primary goal of JUNO is to determine the mass ordering of the neutrinos. JUNO's Central Detector (CD) is a Liquid Scintillator (LS) detector enclosed in an acrylic sphere with an inner diameter of $35.4 \mathrm{~m}$ and a wall thickness of $12 \mathrm{~cm}$. Ultrapure water will be filled outside of the acrylic sphere, in which region there will be 17000 20-inch and 25000 3-inch photomultipliers (PMTs) viewing the scintillation photons produced by the inversed beta decay events occurred inside $\mathrm{CD}$ when electron anti-neutrinos interact with the protons in the LS. In order to determine the neutrino mass hierarchy to more than $3 \sigma$ median sensitivity within 6 years of operation, JUNO detector is required to have very high energy resolution of $3 \% / \sqrt{E}$ and an absolute energy scale uncertainty of less than $1 \%$ [1-5].

Ideally, a large LS detector is a total absorption calorimeter, with its total number of detected photoelectrons proportional to the deposited energy. However, due to geometrical effects and complication in the photon propagation (e.g. internal reflections at the acrylic-water surface), the total photoelectron yield is significantly position dependent, as indicated in Fig. 1 (a), particularly for the region close to the detector boundary. This position non-uniformity, which would otherwise produce an energy bias and an energy-independent constant term to the energy resolution, clearly needs to be calibrated and corrected [2]. To achieve this, the JUNO calibration system consists of four independent systems, each of which is capable of deploying radioactive sources into certain region in the CD, as illustrated in Fig.1 (b). In this paper, we will focus on the design of the Guide Tube Calibration System (GTCS), which brings radioactive source along a chosen longitude along 


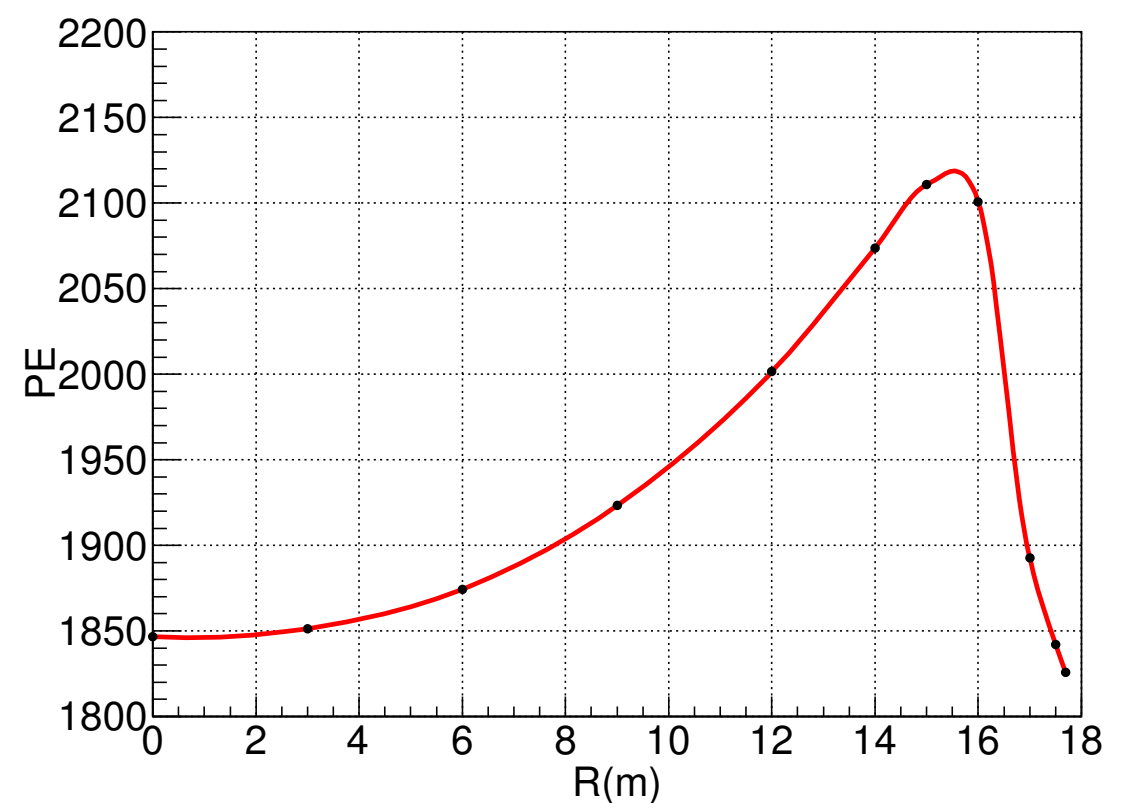

(a)

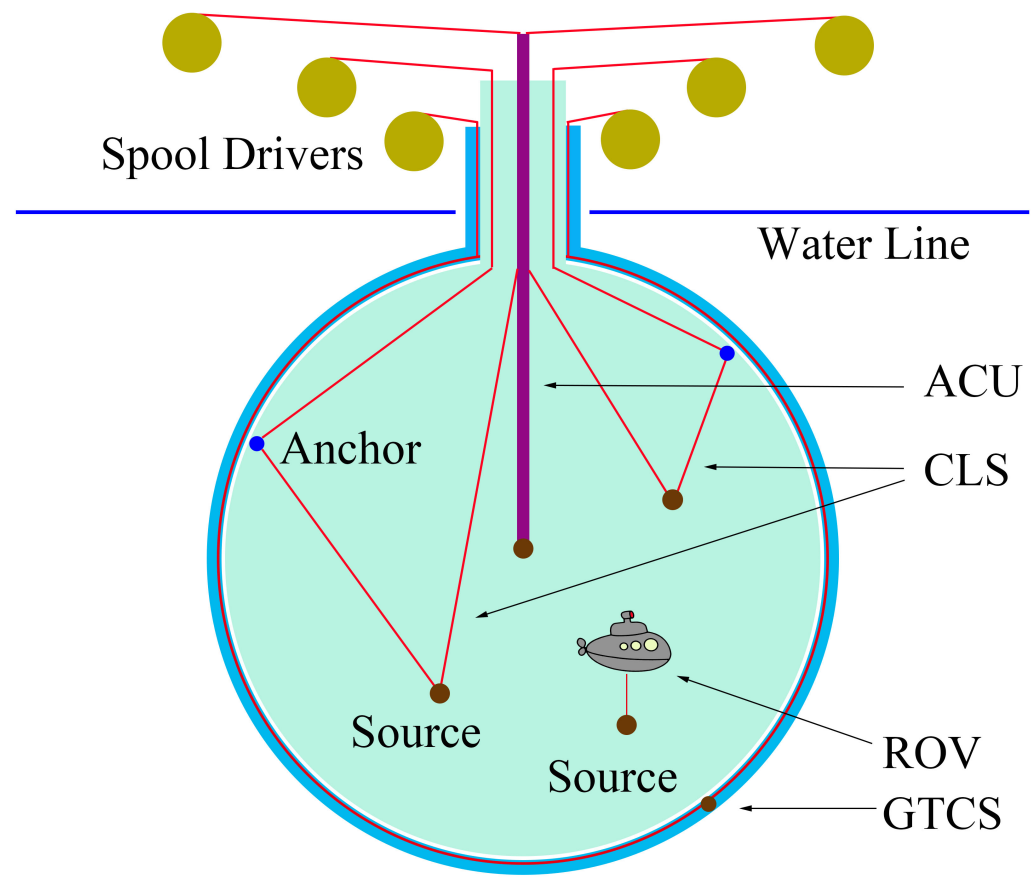

(b)

Figure 1. (a) Non-uniformity of the photoelectron yield of the JUNO CD as a function of radius, simulated with $1.5 \mathrm{MeV}$ electrons. (b) Diagram of JUNO Calibration System including the Automatic Calibration Unit (ACU), the Cable Loop System (CLS), the Guide Tube Calibration System (GTCS) and the RemotelyOperated Vehicle (ROV). 
the CD boundary. Similar calibration systems are used in experiments such as Double Chooz [6] and CUORE [7], but the detector size of JUNO clearly poses additional design challenges.

The rest of this paper is organized as follows. In the next section, the boundary response of JUNO will be thoroughly studied by simulation, based on which we settle on the location of GTCS and the calibration strategy. Then in Section 3, we discuss the design of the system as well as some tests with a prototype setup.

\section{Physics Considerations of the GTCS Design}

As mentioned in the introduction, JUNO requires an overall absolute energy scale uncertainty of $1 \%$. We therefore require that the systematic uncertainty of the energy scale calibration at the detector boundary by GTCS shall reach the same level of $1 \%$. In this section, we will discuss simulation studies connecting this basic requirements to the mechanical design of GTCS. For simplicity, in this paper we discuss the study using a ${ }^{40} \mathrm{~K}$ gamma source $(\sim 1.46 \mathrm{MeV}$ single gamma ray) for which the location of the full absorption peak is used to calibrate the energy scale, but our conclusion remains unchanged if other gamma or neutron sources are considered.

\subsection{Outside or Inside CD?}

To mock up a real physics events happening at the detector boundary, ideally one would like to deploy a gamma source along the inner surface of the acrylic sphere. However, for mechanical reasons and cleanliness considerations, deploying the source along a tube on the outer surface of acrylic is much more feasible. For a nominal gamma source, the latter option will inevitably lead to a stronger Compton leakage tail. Therefore the first important issue to study is the systematic difference in the full absorption peaks in these two options.

The simulation was based on the JUNO offline simulation software SNIPER with version J17v1r1-pre1, a Geant4-based simulation framework $[9,10]$. To accurately simulate the detector response at the $\mathrm{CD}$ boundary, realistic engineering geometry has been incorporated into the SNIPER. The JUNO CD is immersed in water. The acrylic sphere is mechanically held by the Stainless-steel (SS) latticed Shell with 591 SS connection bars. These bars are distributed along 23 different latitude lines [8], and connected to the acrylic nodes on the acrylic sphere. A SS fastener (SF) is embedded inside each acrylic node to hold the bar. On top of the $\mathrm{CD}$, a $800 \mathrm{~mm}$ penetration mates to a SS chimney connecting to the calibration house and the LS filling system. Optical properties of these "dead" components are modeled based on data from bench measurements. We assume that the guide tube is a reflective PTFE tube with a reflectivity of $90 \%$, an inner diameter of $16 \mathrm{~mm}$ and a thickness of $1.5 \mathrm{~mm}$.

The typical total photoelectron (PE) spectrum from a ${ }^{40} \mathrm{~K}$ source located in a guide tube attached to the outer CD surface is shown in Fig. 2 (black histogram). The full absorption peak is clearly observed, but overlaps with the Compton leakage tail. For comparison, the spectrum corresponding to a naked ${ }^{40} \mathrm{~K}$ source located at the $\mathrm{CD}$ inner surface is overlaid in the same figure (blue histogram). In this case the contribution from the tail is reduced by a factor of two or so, but still significant, as expected. The CD is only sensitive to the converted gamma energy in the active LS region, therefore whether the gamma originates from the inner or outer surface does not make a great difference as in either case there is significant inactive volume surrounding the gamma vertex. 


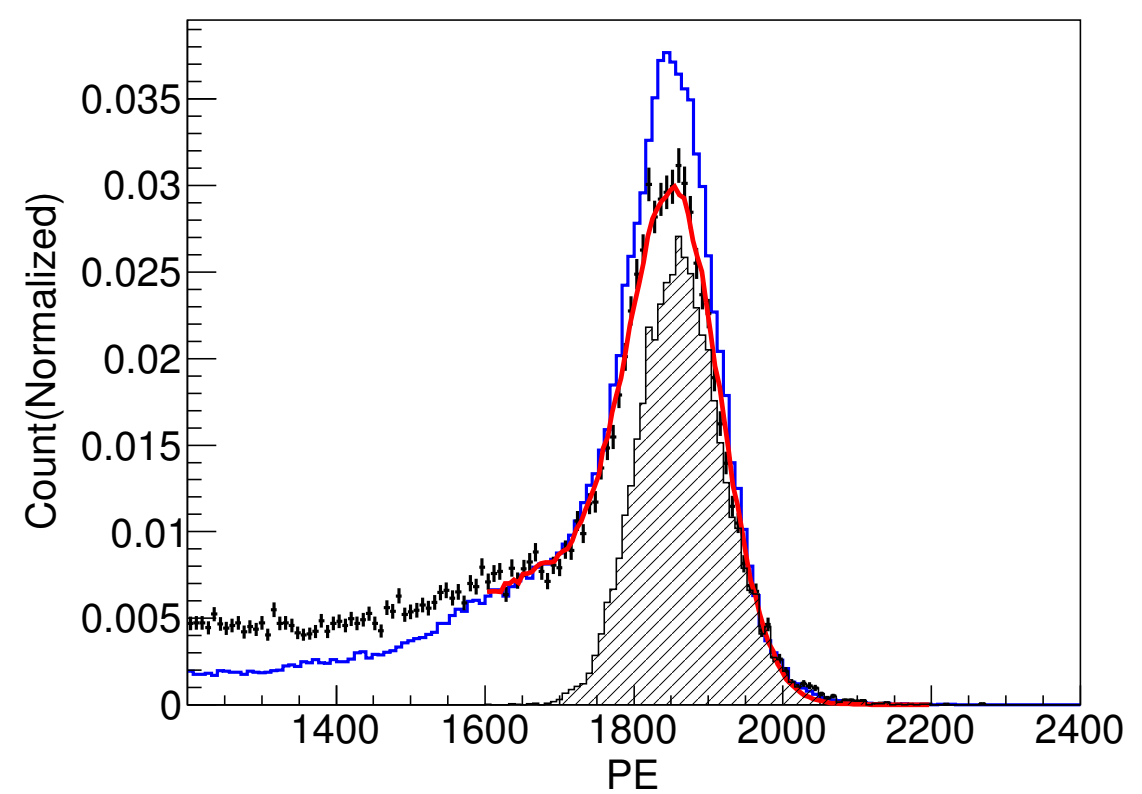

(a)

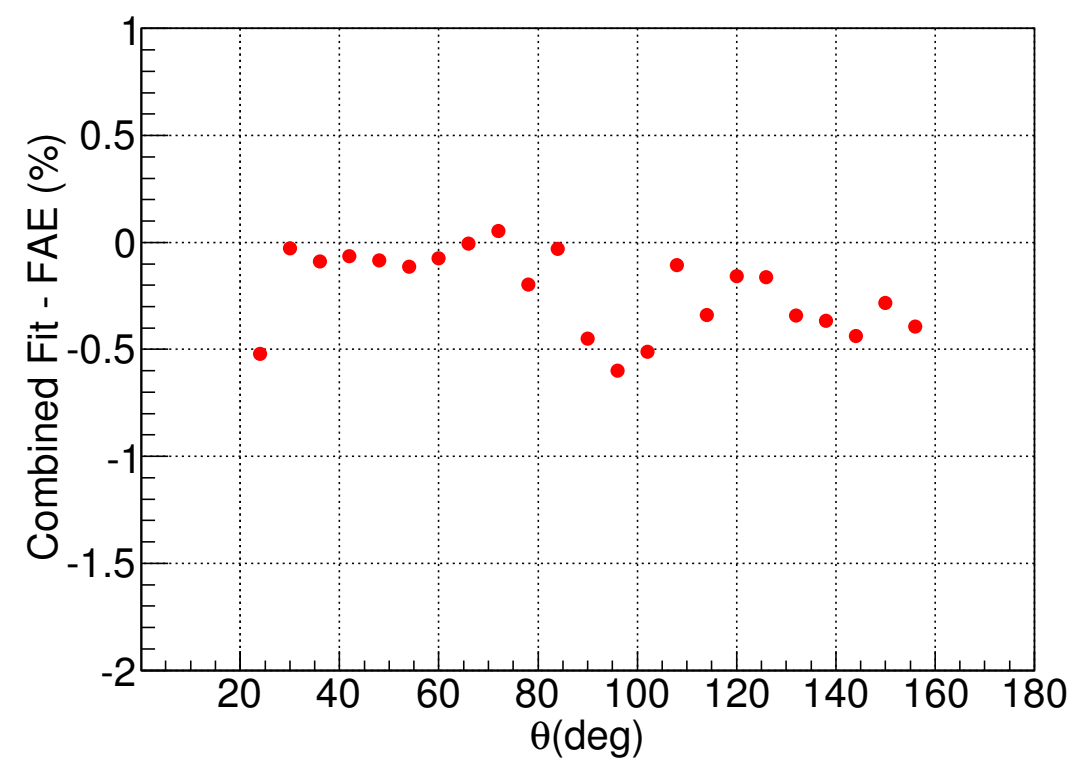

(b)

Figure 2. (a) Black: The typical PE spectrum from a ${ }^{40} K$ source received by PMTs. Shaded: The corresponded FAE peak. Red: The fitting result with $\mathrm{Eq}(2.1)$. Blue: The PE spectrum corresponding to a naked ${ }^{40} \mathrm{~K}$ source located at $\mathrm{CD}$ inner surface. (b) The fractional difference between the combined fitted Gaussian peak and the true FAE peak, indicating the uncertainty of the combined fit. 
We fit the observed spectrum by using a combination of a full absorption peak and Compton tail as shown in $\mathrm{Eq}(2.1)$,

$$
f_{\mathrm{m}}(\mathrm{PE})=\mathrm{g}(\mu, \sigma)+\mathrm{f}_{\mathrm{c}}(\mathrm{PE}),
$$

where $g(\mu, \sigma)$ is a Gaussian function with mean $\mu$ and standard deviation $\sigma$, and $f_{\mathrm{c}}(\mathrm{PE})$ is an average Compton tail (over an entire latitude line) obtained from simulation, with the vertical scale floating in the fit. It was verified that for all locations such a model can produce satisfactory fit. For a nominal 30000 calibration events, the statistical uncertainty of the centroid of the Gaussian reaches $0.1 \%$. In the simulation, we can also select fully absorbed events (FAE) with all gamma energy deposited in the active region. To evaluate the systematic uncertainty from the combined fit, the centroid in Fig. 2 (a) is compared to that for the FAEs for different locations on the latitude (Fig. 2 (b)). Due to the CD top chimney and bottom flange affecting tube deployment, which will be mentioned in section 3.2 in details, only the points in the range from $24^{\circ}$ to $156^{\circ}$ are studied. On average, one observes a scattered difference with a range of $\pm 0.6 \%$, which serves as an estimate of systematic uncertainty of such fit.

Beside the fit systematics, the mean values of the FAE for the source located at the inner or outer CD surface is also compared in Fig. 3 as a function of the azimuthal angle. One sees that on average the inner response (ideal) would be about $0.3 \%$ higher than that for the guide tube attached on the outer surface. Such a bias is, in principle, correctable based on simulation. However, for conservativeness, we take it also as a source of systematic uncertainty. Therefore, the overall systematic uncertainty of the FAE peak when the source is located at the outer surface of the CD is $\sqrt{0.6^{2}+0.3^{2}} \approx 0.7 \%$.

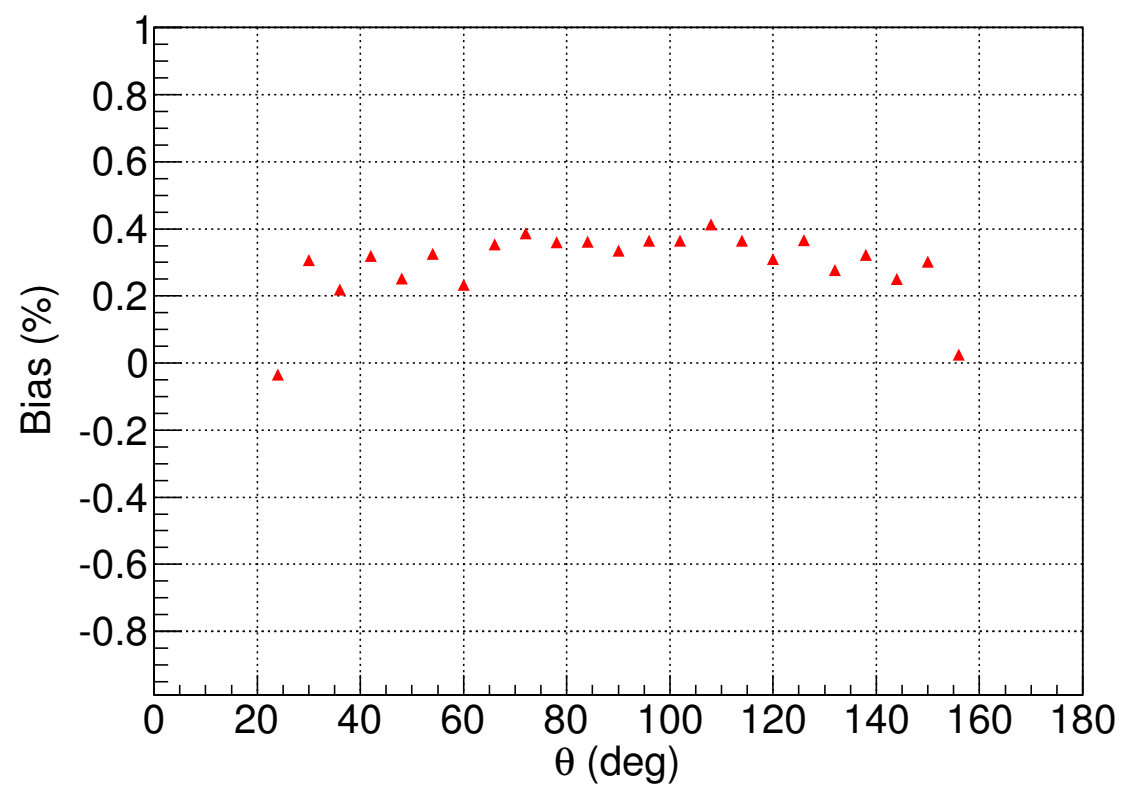

Figure 3. The relative difference between the FAEs when the source in is located at the inner (ideal) or outer (practical) surface of the $\mathrm{CD}$.

To conclude from the studies above, the systematic uncertainty to the FAE for the outer GTCS 
strategy is estimated to be $0.7 \%$. This is satisfactory in comparison to the sub- $1 \%$ requirement for the total energy scale uncertainty. We therefore settle on this option as the baseline design of GTCS.

\subsection{Choosing the Path of the Guide Tube}

As mentioned above, for the region close to the CD boundary, many light blocking structures exist, such as the SF. To settle on the location of the GTCS, several representative scans with steps of $1^{\circ}$ were carried out along the longitude line of $\phi=0^{\circ}, \phi=4^{\circ}, \phi=7^{\circ}$ and the latitude line of $\theta=84^{\circ}, 90^{\circ}$, respectively. The results are shown in Fig. 4. Three structures affect the response significantly. The SS chimney has significant shadowing effects, which causes the steep slope on the left side of longitude scan response curve in Fig. 4 (a). The SF causes a strong local light blocking effect in the area close to the SF (strong-SF area), as shown in Fig. 4 (a) with $\phi=0^{\circ}$ and $7^{\circ}$. Even at locations further away, some residual structure can be observed (weak-SF area). This is obviously demonstrated in the latitude response curves in Fig. 4 (b) with $\theta=84^{\circ}$ and $90^{\circ}$, as well as in Fig. 4 (a) with $\phi=4^{\circ}$. In addition, the photon reflection on the guide tube also affect the detector's response.

From the mechanical point of view, moving a source along the longitude is also easier in comparison to along the latitude, e.g. around the equator, due to a smaller friction between the source assembly and the tube. More importantly, since the SF placement exhibits some symmetry in $\phi$ (so is the chimney), the GTCS should be installed along the longitude, as long as the calibration along this line would reflect the $\phi$-averaged response. For this reason, this line should not be too close to the SF, otherwise local light blocking effects would be overly emphasized and bias the overall response. The goal of the following study is to find out an efficient way to obtain the average response function at the $\mathrm{CD}$ boundary.

To do this, we selected eleven longitude lines in the weak-SF area $\left(\theta=30^{\circ}, 42^{\circ}, 54^{\circ}, 66^{\circ}, 78^{\circ}\right.$, $90^{\circ}, 102^{\circ}, 114^{\circ}, 126^{\circ}, 138^{\circ}, 150^{\circ}$ ), assuming the PTFE tube's reflectivity is $90 \%$, and simulated the detector response. In addition, we also obtained a $\phi$-averaged response using a perfect source from the simulation. The comparison of these response curves is made in Fig. 5 (a). The residual difference between "calibration data" and the average is shown in Fig. 5 (b). The overall difference is less than $0.4 \%$. We also applied different reflectivities in the studies and the same conclusion is reached.

As a result, the guide tube could be installed along any longitude line as long as the route is not blocked by a SF structure. The accurate position will be given in next chapter with the consideration of CD surface structures.

\subsection{Estimation of Uncertainty}

The uncertainty of the energy scale by GTCS can be classified into four sources: a) statistic, b) FAE peak fitting, c) $\phi$-averaged function reconstruction and d) source positioning uncertainty, in which b) and c) have been discussed in Secs. 2.1 and 2.2, respectively. For a), we plan to use a $1000 \mathrm{~Bq}$ ${ }^{40} \mathrm{~K}$ source, which produced approximately $100 / \mathrm{s} 1.46 \mathrm{MeV} \gamma$-ray. At the GTCS location, about $5 \%$ of the events would be FAEs. Assuming a 5-minute nominal data taking period at each source location, the statistical uncertainty of the FAE is estimated to be $0.1 \%$, according to Fig. 2 .

To address d), we expect that the vertex reconstruction uncertainty by JUNO photomultipliers can reach a level of $10 \mathrm{~cm}$ for $\mathrm{MeV}$-scale positron events. Therefore, we have required that all 


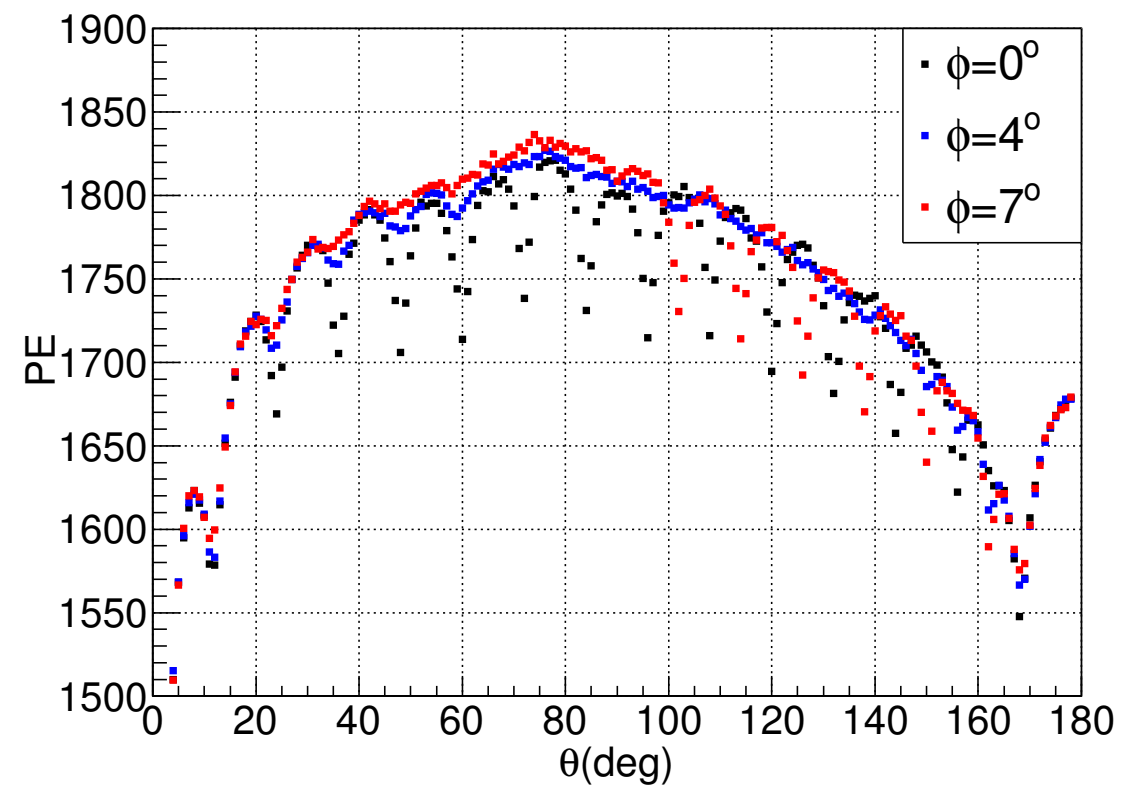

(a)

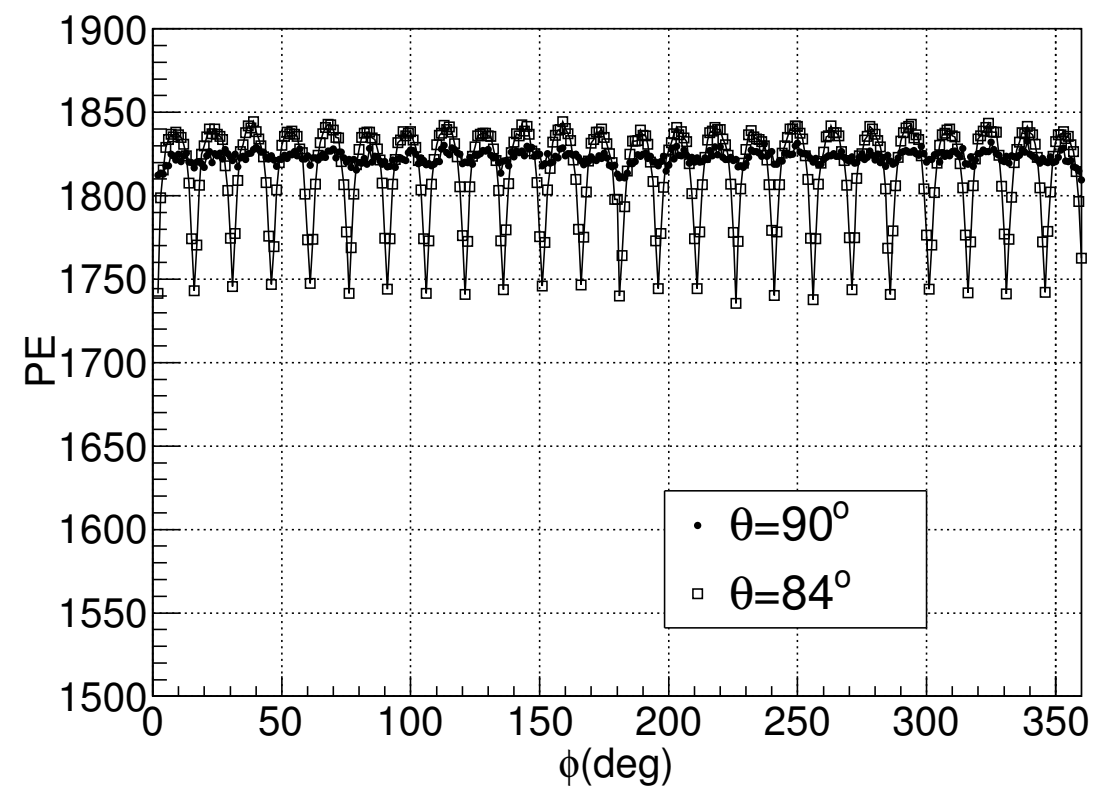

(b)

Figure 4. Longitude scan (a) and latitude scan (b) results. 


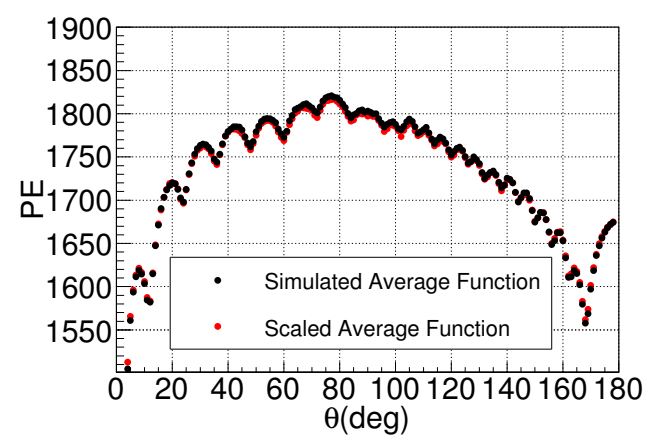

(a)

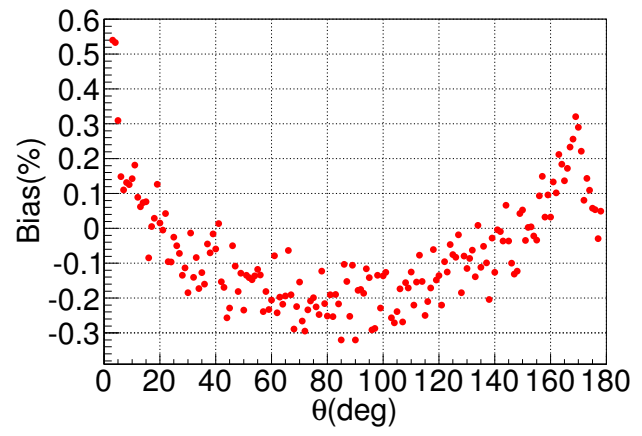

(b)

Figure 5. (a) The black points are the average response function acquired from the naked source simulation and the red points are the scaled response function along a longitude line. (b) The residual difference in fraction between the two in (a).

source locations should be measured (independent of the reconstruction by photomultipliers) after installation. To study the impact of positional uncertainty on the GTCS response, source positions were shifted intentionally between $3 \mathrm{~cm}$ and $10 \mathrm{~cm}$ with respect to the nominal calibration positions, and the resulting bias to the energy response function was built using these biased positions, as shown in Fig. 6. One observes that the maximum bias is at $0.04 \%$ level for a positional bias of $3 \mathrm{~cm}$, and increases to $0.15 \%$ level for a bias of $10 \mathrm{~cm}$. The latter bias is used as a conservative uncertainty due to the GTCS positioning uncertainty.

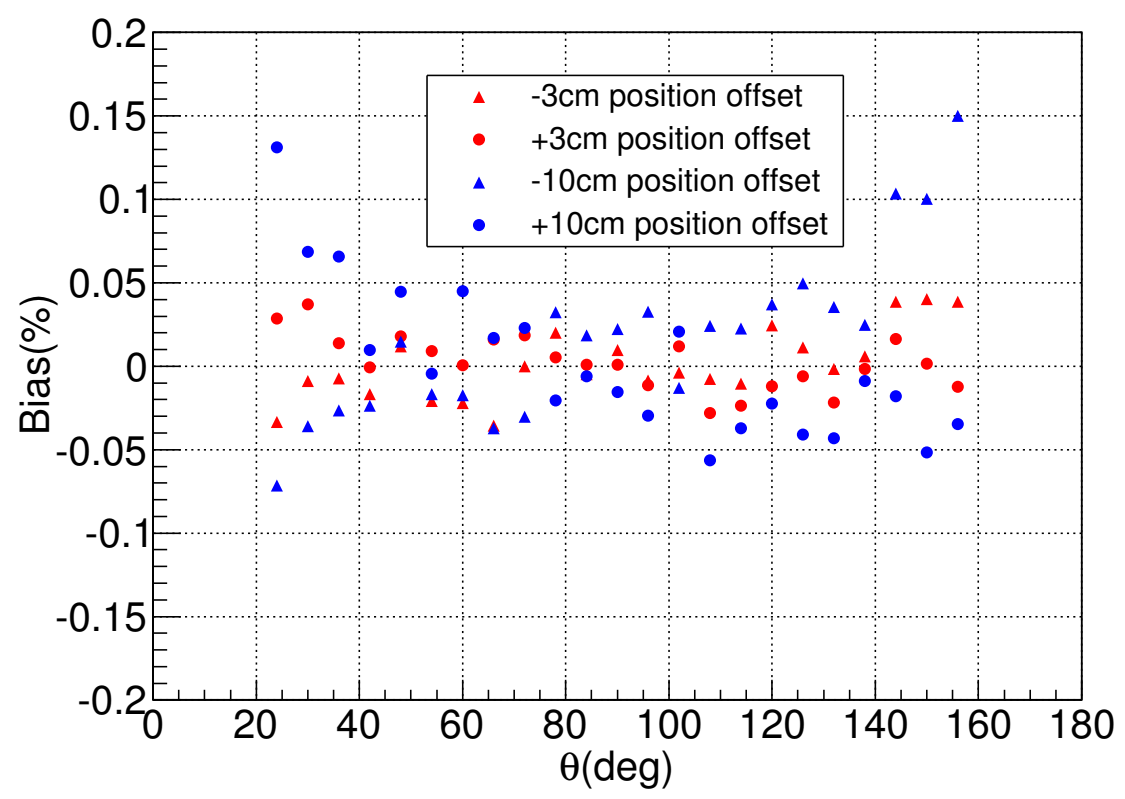

Figure 6. Response bias due to different positioning bias of the GTCS source.

As a summary, all four uncertainty sources of the GTCS is given in Table 1. The overall 
uncertainty of the energy scale is estimated to be $0.83 \%$, which meets the $1 \%$ requirement.

Table 1. Uncertainty estimation of GTCS energy response.

\begin{tabular}{|c|c|c|c|c|}
\hline Statistical & Positional bias & FAE fitting & Average response reconstruction & Total \\
\hline $0.1 \%$ & $0.15 \%$ & $0.7 \%$ & $0.4 \%$ & $0.83 \%$ \\
\hline
\end{tabular}

\section{GTCS Mechanical Design}

Based on the study above, we now discuss the mechanical design of the GTCS. Our primary purpose is to deliver the radiation source to the designated positions and later to retract it safely. The system is divided into three parts: (1) the source and cable assembly; (2) guide tube and its fixing anchors on the CD; (3) winding machine and its control system. A general diagram of GTCS is shown in Fig. 7.

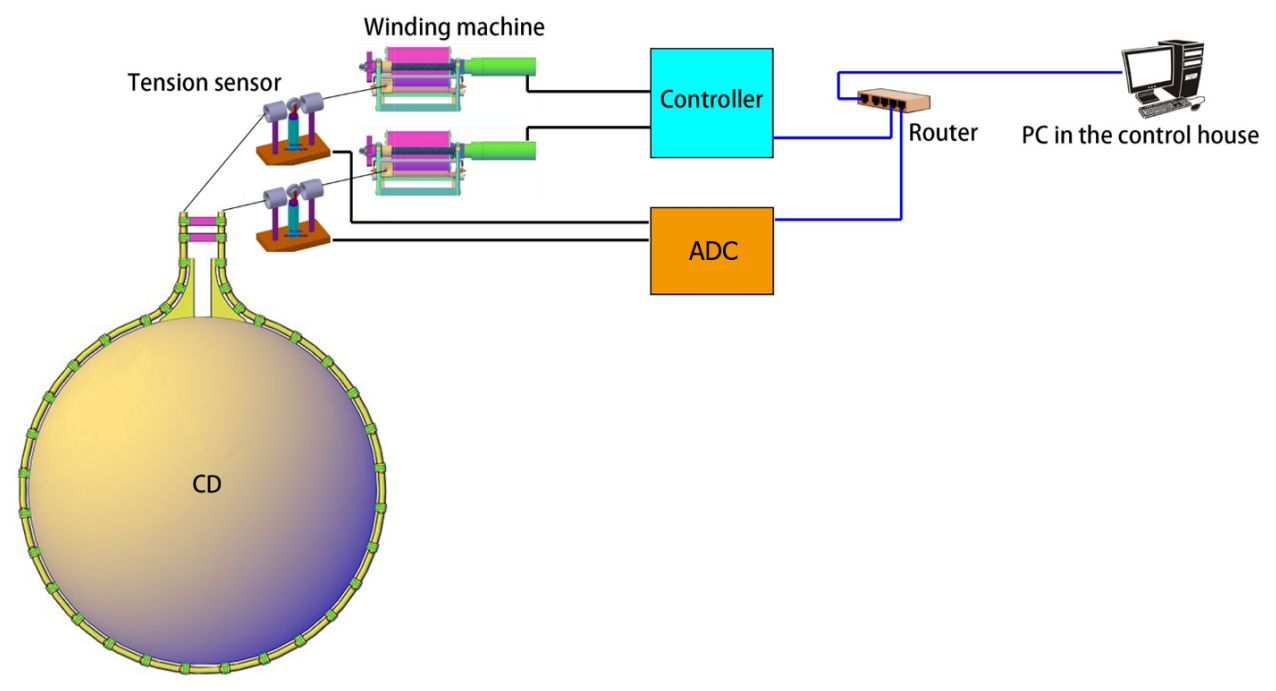

Figure 7. A general diagram of GTCS.

\subsection{Source and Cable Assembly}

The design of the radioactive source assembly is illustrated in Fig. 8. The calibration source will be sealed in a SS cylinder $(\phi 6 \times 6)$. The enclosure of the cylinder is made out of a PTFE cylinder with an overall dimension of $\phi 13 \times 20.5$, in order to minimize friction and turning radius. Two 130-m-long cables are attached on both ends of it to move the source assembly in two directions. This design also offers extra insurance if either the source is stuck in the tube or one cable is broken. The cable is a SS wire with a diameter of $0.7 \mathrm{~mm}$ coated by a 0.15 -mm-thick FEP. A tensile test indicated its minimum breaking force is greater than $800 \mathrm{~N}$. 


\section{0

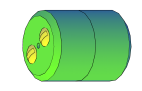

Figure 8. The design of the source assembly.

\subsection{The Tube and its Fixing Anchors}

The main body of GTCS is a 130-m-long PTFE tube with inner diameter of $16 \mathrm{~mm}$ and a wall thickness of $1.5 \mathrm{~mm}$. PTFE is chosen to minimize friction. The tube should be deployed along a longitude of acrylic sphere and then go along the JUNO chimney to get out of the water shield.

The fixture of the tube on CD surface is also called the GT anchor. As shown in Fig. 9, it is composed of three parts. The dark blue part is an acrylic base and it will be bonded onto the CD surface via polymerization bonding method. The shape is carefully designed to minimize residual stress during bonding. Both the cyan and grey parts are adjustable nylon holder for the tube. The tube will go through the hole in grey holder which will be fixed on the cyan holder using bolts.

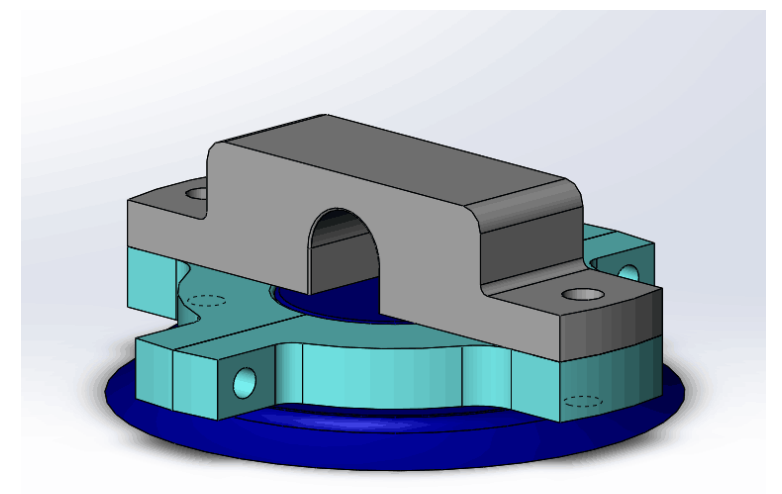

Figure 9. The design of the GT anchor outside the CD.

On the $\mathrm{CD}$, there are in total 58 anchors, about one in every two meters. Near the CD chimney, a turning track with a radius of $2 \mathrm{~m}$ made of SS tube is fixed on the SS connection bars with rods, as shown in Fig. 10.

As discussed in Section 2.2, the guide tube could be installed along any weak-SF longitude line to represent a $\phi$-averaged boundary response. To avoid conflict with the SF structure, the whole GT circle is divided into two half circles: one is on the longitude of $2.61^{\circ}$ and the other one is on the longitude of $146.61^{\circ}$, as illustrated in Fig. 11. At the bottom of CD, the PTFE tube detours smoothly from the bottom flange of the CD. 


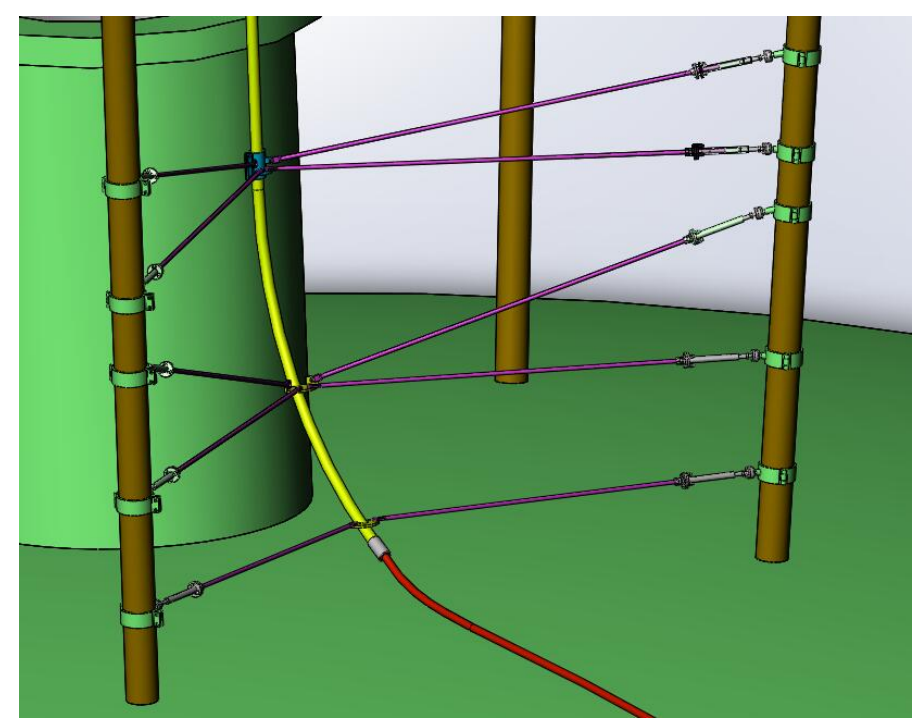

Figure 10. Details of the turning track for the GT.

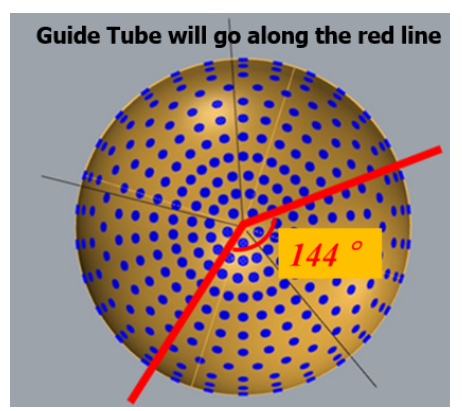

(a)

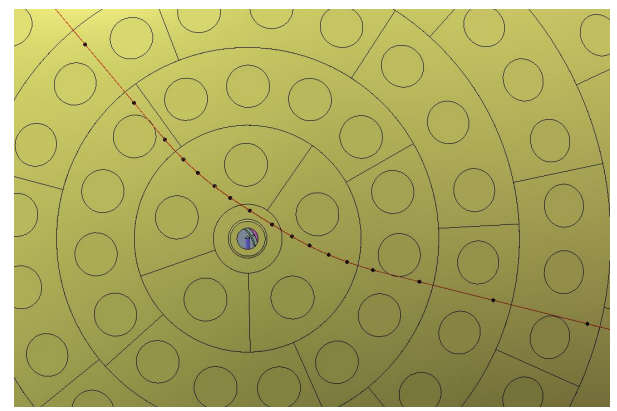

(b)

Figure 11. (a) The GT and its anchors on the CD surface; (b) the routing of the GT close to the bottom of the CD. The SF placement has a 5-fold symmetry in $\phi$ and the $\phi$ angle between two half circles of GT is $144^{\circ}$.

The GT will be immersed in the ultrapure water shield with a maximum height of $40 \mathrm{~m}$. To avoid deformation due to water pressure, the guide tube is designed to be filled with water, which will be recirculated to ensure the cleanliness.

\subsection{Winding Machine and its Control System}

The winding machine is the key part of GTCS. It will control the source assembly position by dragging the deployment cables, as shown in Figs. 12 (a) and (b). Its main body is a PTFE wire spool with helical grooves on which the cable is wound in order. A servo motor, controlled by a LabVIEW software, drives the spool automatically. The tension in the cable is monitored constantly to avoid the source getting caught unexpectedly in the GT. To achieve this, a load cell is mounted below a pulley through which the cable goes through with an angle of $120^{\circ}$ before the spool (please see Fig. 12 (c)).

The winding spool obtains the source location via the length of cables. Although guide tube 


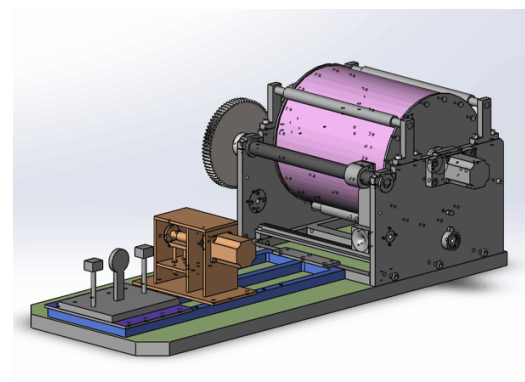

(a)

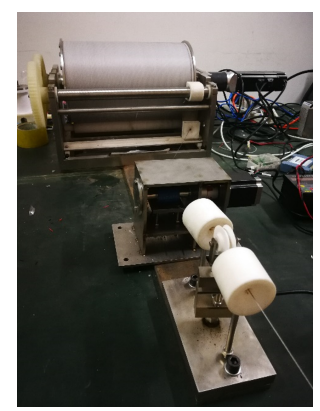

(b)

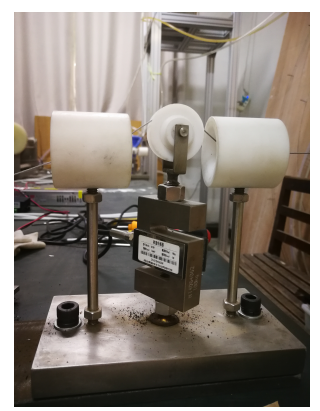

(c)

Figure 12. Design drawings of the winding machine (a) and (b), and the tension pulley (c).

is fixed onto the anchors approximately every two meters, the distortion of the guide tube could still introduce a positioning uncertainty. To better control this, metal proximity sensors will be installed close to a few selected CD anchors. A signal will be triggered when the source assembly goes by a sensor, with a sub-cm precision based on a prototype test. With these sensors, the source positioning uncertainty for the entire GT loop is expected to reach the $3-\mathrm{cm}$ requirement.

The winding machines and related auxiliaries will be enclosed in a GTCS box, as shown in Fig. 13, which is located on a bridge above the water and close to the chimney. To avoid light leakage into the water and support the guide tude vertically, a SS tube enclosing the guide tube above the water is connected to turning track and goes to the GTCS box with a sealed penetration.

Our mechanical design was verified under realistic conditions. While details of this test will be reported elsewhere, we comment on the general performance here. We constructed a full-size circular track, horizontally laid on the ground with a diameter of $35.4 \mathrm{~m}$. The guide tube was attached to the track. The motion of the source assembly was automatically controlled and tested, showing the required precision could be satisfied. The maximum friction between source assembly and tube was measured to be less than $20 \mathrm{~N}$, which is very low compared to the break strength of $800 \mathrm{~N}$.

\section{Summary}

In summary, we have designed a guide tube calibration system for the JUNO experiment. Based on a realistic simulation, such a system is capable of calibrating the energy scale at the CD boundary to a sub-percent level. The mechanical design of the GTCS has beenm developed and verified to full scale.

\section{Acknowledgments}

We thank Tao Zhang from SJTU, Xiaoyan Ma and Xiaohui Qian from IHEP for engineering support in the mechanical design. This research is supported by the "Strategic Priority Research Program" of the Chinese Academy of Sciences (Grant No. XDA10000000). 


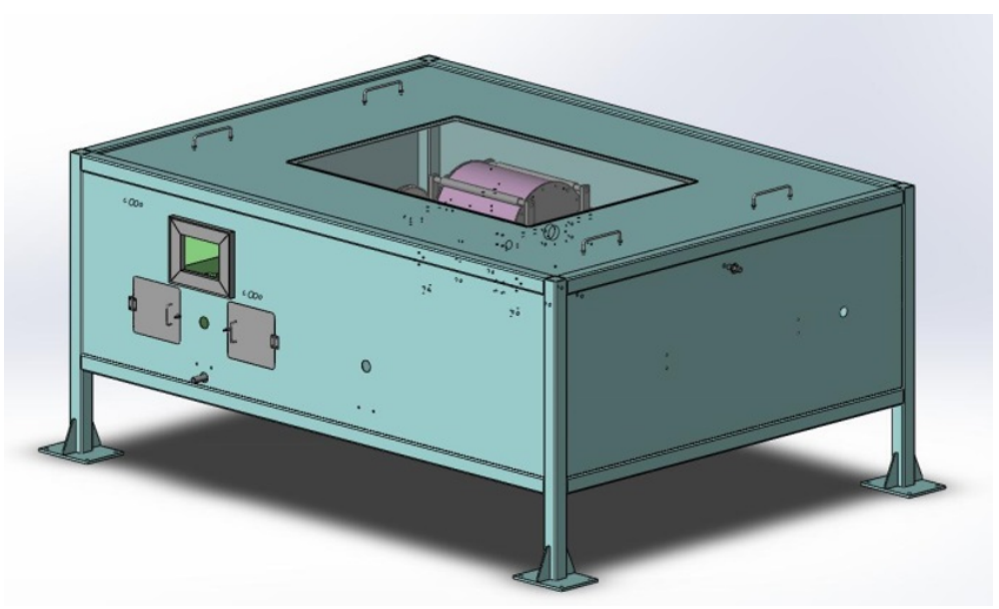

(a)

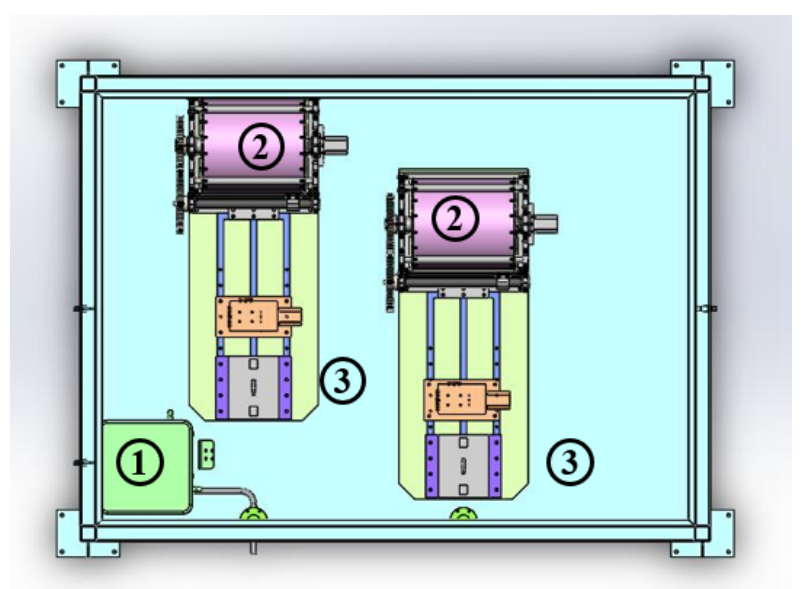

(b)

Figure 13. (a) External appearance of the GTCS box. (b) Internal layout of GTCS box: (1) tank of the water recirculating system (2)winding machines (3) cable tension pulleys.).

\section{References}

[1] Adam, T. et al., JUNO Conceptual Design Report., arXiv:1508.07166

[2] An, Fengpeng et al., Neutrino Physics with JUNO, JOURNAL OF PHYSICS G-NUCLEAR AND PARTICLE PHYSICS, 2015, 43(3).

[3] Y. F. Li et al., Unambiguous Determination of the Neutrino Mass Hierarchy Using Reactor Neutrinos, Phys. Rev. D 88, 013008(2013).

[4] Liang Zhan et al,Experimental requirements to determine the neutrino mass hierarchy using reactor neutrinos, Physical Review D 79, 073007(2009), arXiv:0901.2976.

[5] Qian X et al, Mass Hierarchy Resolution in Reactor Anti-neutrino Experiments: Parameter Degeneracies and Detector Energy Response, Physical Review D, 2012, 87(3):691-695.

[6] Igor Ostrovskiy et al, Double Chooz Calibration, Nuclear Physics B (Proc. Suppl.) 229âĂŞ232 (2012) 431 Double. 
[7] Cushman J S et al. The detector calibration system for the CUORE cryogenic bolometer array. Nuclear Inst \& Methods in Physics Research A, 2017, 844:32-44.

[8] WANG YuanQing et al, Application of an acrylic vessel supported by a stainless-steel truss for the JUNO central detector, Science China Technological Sciences,2014,57(12),2523-2529.

[9] Zou J H et al, SNiPER: an offline software framework for non-collider physics experiments, Journal of Physics: Conference Series 664 (2015) 072053, doi:10.1088/1742-6596/664/7/072053.

[10] Allison, John, Facilities and Methods: Geant4-A Simulation Toolkit, Nuclear Physics News, 17:2, 20-24(2007). 